\title{
Cerebral Arteritis With Extremely Late Onset Secondary to Bacterial Meningitis
}

\author{
-Case Report-
}

\author{
Tomohiro KaWAgUCHI, ${ }^{1,2}$ Yoshikazu OGAWA, ${ }^{1}$ \\ Takashi INOUE, ${ }^{1}$ and Teiji TOMINAGA ${ }^{2}$
}

\author{
${ }^{1}$ Department of Neurosurgery, Kohnan Hospital, Sendai, Miyagi; \\ ${ }^{2}$ Department of Neurosurgery, Tohoku University Graduate School of Medicine, Sendai, Miyagi
}

\begin{abstract}
A 59-year-old woman presented with bacterial meningitis causing arterial narrowing in the very late stage. She initially underwent transsphenoidal surgery for massive non-functioning pituitary adenoma resulting in gross total removal. The postoperative course was uneventful, and she was discharged 12 days after surgery. Fourteen months later, she presented with bacterial meningitis due to streptococcus. Administration of multiple antibiotics resulted in normalization of cerebrospinal fluid findings. Forty days after meningitis onset, she suddenly suffered motor weakness of the right extremities. Magnetic resonance (MR) angiography revealed multiple intracranial arterial narrowings. Despite intensive treatment, no improvement of arterial narrowing was seen, and she suffered cerebral infarction. Six months after the onset of meningitis, MR angiography still showed multiple arterial narrowings, and MR plaque imaging of the stenotic vessel disclosed thickened arterial wall. This case illustrates the complex time course of arteritis caused by severe bacterial meningitis, and the intractability to treatment.
\end{abstract}

Key words: bacterial meningitis, arteritis, vasospasm, plaque imaging, late onset

\section{Introduction}

Bacterial meningitis still occurs with non-negligible morbidity/mortality rates despite the improvement of antimicrobial therapy during the last decades, and unfavorable outcome occurs in $34 \%$ of patients. ${ }^{21)}$ Such unfavorable clinical outcomes are often due to intracranial complications such as brain edema, hydrocephalus, and cerebrovascular complications. Cerebrovascular complications occur in $37 \%$ of patients with bacterial meningitis. ${ }^{14)}$ Several different types of cerebrovascular changes are known to occur after bacterial meningitis, including narrowing of the large arteries, caliber changes in the small to medium arteries, and sinus thrombosis. ${ }^{8,14,18)}$ Caliber change in all sizes of cerebral arteries is the most common cause of ischemic stroke, but this phenomenon includes at least two entities, vasospasm ${ }^{3,22)}$ and arteritis, which must be distinguished because the treatment, clinical course, and outcome are different. The onset, duration, and recommended treatment for vasospasm are well known. However, arteritis is relatively rare with only a few sporadic cases reported, so that the etiology and clinical course are still unknown.

We report a case of bacterial meningitis associated with development of arterial narrowing 40 days after the onset.

Received May 31, 2010; Accepted August 19, 2010

\section{Case Report}

A 59-year-old woman was introduced to the outpatient department of Kohnan Hospital for the treatment of massive non-functioning pituitary adenoma manifesting as bitemporal hemianopsia (Fig. 1A). Magnetic resonance (MR) angiography showed no abnormal features except for elevation of bilateral anterior cerebral arteries (Fig. 1C). Preoperative systemic examination showed no electrocardiographic abnormalities and no cardiac murmur was pointed out. The tumor was gross totally removed through the transsphenoidal approach. The sellar floor was reconstructed with autologous bone and muscle pieces without using fibrin glue or other artificial materials. Cerebrospinal fluid (CSF) leakage was not observed throughout the operation. The postoperative course was uneventful and she was discharged 12 days after surgery (Fig. 1B).

Fourteen months later, she presented with high fever, drowsiness, and neck stiffness. Blood examination demonstrated a white blood cell count of $23800 / \mu \mathrm{l}$ and elevated C-reactive protein (over $35.0 \mathrm{mg} / \mathrm{dl}$ ). CSF contained $131 \mathrm{cells} / \mu \mathrm{l}$, elevated protein content $(270 \mathrm{mg} / \mathrm{dl})$, and decreased glucose concentration (14 mg/dl). Culture of CSF demonstrated Streptococcus species. Based on these findings, the diagnosis was bacterial meningitis. Simultaneous administration of multiple antibiotic agents 



Fig. 1 A: Preoperative $T_{1}$-weighted magnetic resonance (MR) image with contrast medium showing an intra- to suprasellar mass lesion compressing the optic chiasm. B: Postoperative $\mathrm{T}_{1}$-weighted MR image showing that the mass lesion was totally removed. C: Preoperative MR angiogram showing no vascular abnormality.
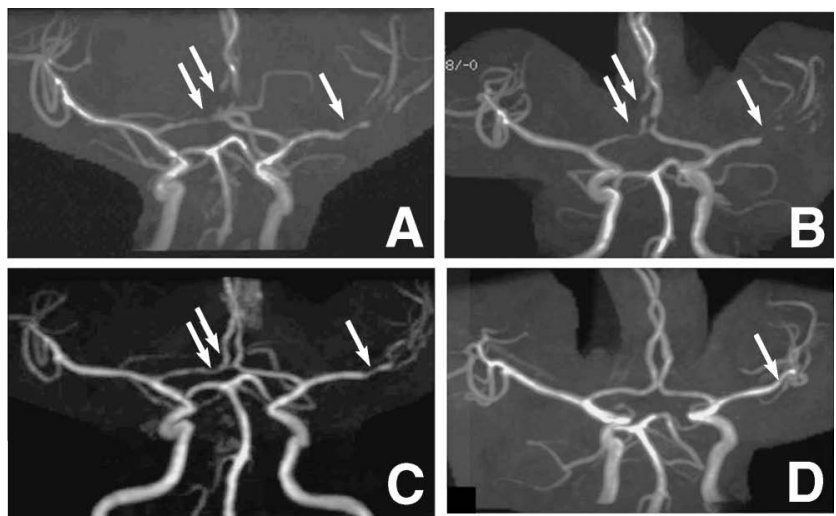

Fig. 2 A: Magnetic resonance (MR) angiogram taken after the occurrence of right hemiparesis showing multiple arterial narrowings (arrows). B, C: MR angiograms at 3 (B) and 6 months (C) after meningitis onset showing slight improvement of arterial caliber, but persistence of narrowing (arrows), especially of the left distal middle cerebral artery. D: MR angiogram at 21 months after meningitis onset showing nearly complete resolution of the arterial caliber of the left middle cerebral artery (arrow).

resulted in normalization of CSF findings, blood examinations, and vital signs without focal deficits, and she was almost ready for discharge on foot.

Forty days after meningitis onset, she suddenly present-
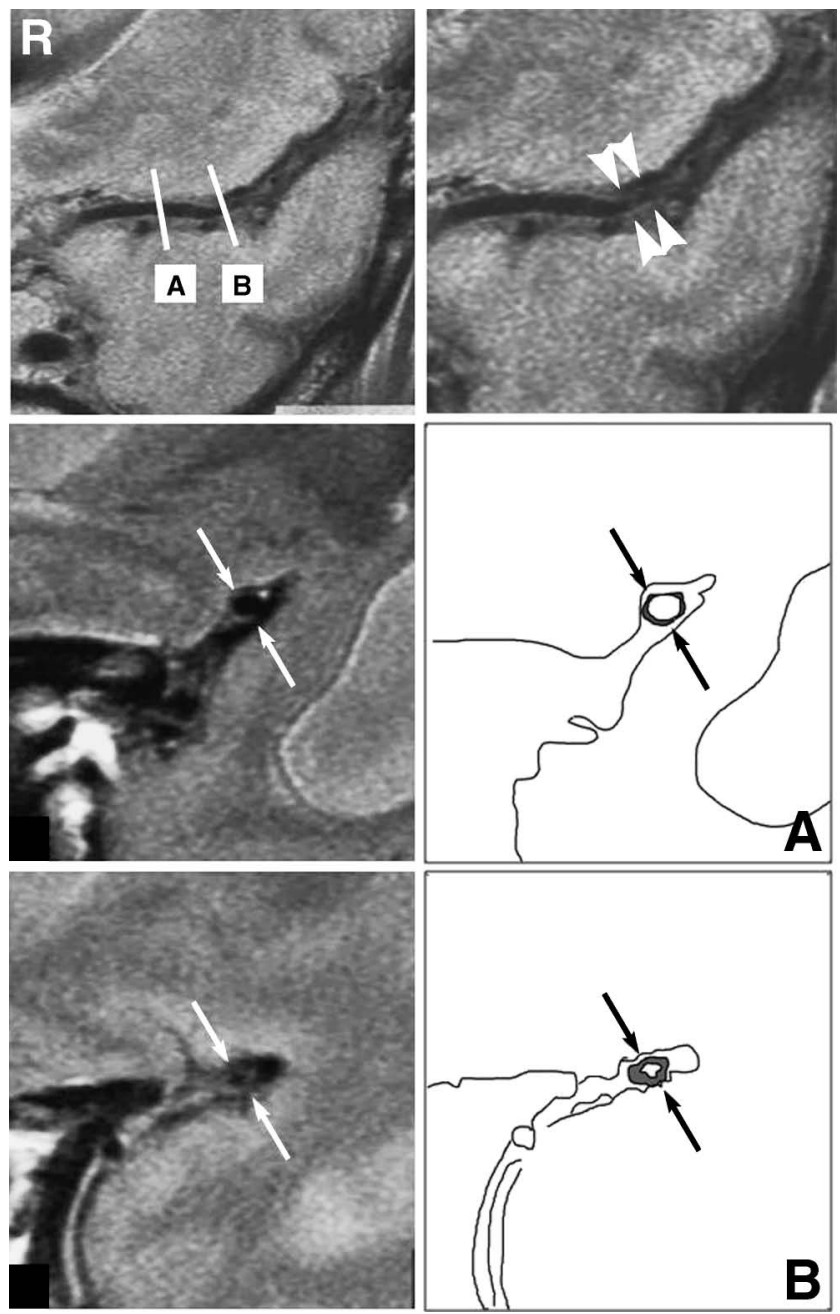

Fig. 3 Magnetic resonance (MR) plaque images of longitudinal slices along the left $M_{1}$ showing arterial narrowing (upper right column, arrowheads). Transaxial images showing no vessel wall thickening in the normal caliber lesion (section $A$, arrows), but the vessel wall was remarkably thickened at the narrow lesion (section $B$, arrows). These thickened walls did not show hyperintensity, contraindicating atherosclerotic change.

ed with slight right hemiparesis (manual muscle test [MMT] 4/5). MR angiography demonstrated multiple arterial narrowings (Fig. 2A). Initially, vasospasm was suspected, so she was treated with antiplatelet agents, hypervolemic therapy, and hyperdynamic therapy. Blood examination showed that both white blood cell count and Creactive protein were within the normal ranges. Lumbar puncture was performed, and CSF examination showed no infectious signs. Since therapy resistant and delayed vasospasm was initially suspected, a spinal drainage tube was introduced for intrathecal administration of nicardipine. ${ }^{19)}$ Moreover, steroid pulse therapy was carried out with $1000 \mathrm{mg} /$ day of hydrocortisone for possible involvement of inflammatory mechanisms. In spite of such intensive treatments, her symptom gradually aggravated and diffusion-weighted MR imaging showed a hyperintense le- 
sion at the left corona radiata. Finally, her hemiparesis became almost plegie (MMT 1/5).

Follow-up MR angiography, which was carried out 3 and 6 months later, showed prolonged arterial narrowing, especially of the left middle cerebral artery (Fig. 2B, C). Six months after the onset, 3-T MR plaque imaging (3T Signa Excite; GE Healthcare, Chalfont St. Giles, Buckinghamshire, UK) using a fast spin echo sequence (repetition time $2800 \mathrm{msec}$, echo time $51.1 \mathrm{msec}$ ) of the left $\mathrm{M}_{1}$ portion of the left middle cerebral artery revealed the thickened wall (Fig. 3). These thickened walls did not show hyperintensity, contraindicating atherosclerotic change. Twentyone months after meningitis onset her MR angiography showed nearly complete resolution of the arterial caliber of the left middle cerebral artery (Fig. 2D). Based on these findings and the clinical time course, the diagnosis was reconsidered to be arteritis. She still had severe right hemiparesis (MMT 1/5) and continued motor rehabilitation.

\section{Discussion}

Arteritis is considered to result from several inflammatory mechanisms, either singly or in combination, including encroachment by inflammatory subarachnoid purulent exudates $^{1,4,11,16)}$ or infiltration of the arterial wall by inflammatory cells with the phenomena of intimal thickening, subintimal infiltration of the arterial wall, arterial wall edema, and infiltration of the whole vessel wall.1,2,4) Polymorphonuclear cell infiltration extending via the Virchow-Robin space to the subintimal regions of the small arteries has been identified in a patient with bacterial meningitis. ${ }^{4)}$ Autopsy is the most reliable confirmation for this entity, ${ }^{5)}$ whereas histological examination has limitations for clinical diagnosis. Correct diagnosis based on the clinical course and other objective findings is essentially required.

Angiographic studies have documented arterial narrowing in patients with bacterial meningitis. ${ }^{6,12)}$ Conventional angiography can identify arterial narrowing, but cannot distinguish arteritis or vasospasm because only the arterial lumen and not the vessel wall is imaged. ${ }^{9)}$ Wall thickening and intramural contrast uptake are frequently shown in patients with active arteritis. ${ }^{10)}$ Therefore, precise evaluation of arterial wall thickness using high resolution MR imaging is the most reliable modality for practical treatment and diagnosis.9) This MR sequence was originally reported for detection of atherosclerotic plaque of intracranial artery, but is also useful to evaluate the arterial wall thickness. In the present case, 3-T MR plaque imaging demonstrated the luminal stenosis and thickened arterial wall without atherosclerotic plaque, which led us to the diagnosis of arteritis.

The present case had the longest reported interval between onset of meningitis and arteritis, and very prolonged neuroimaging abnormalities. Angiographical narrowing usually reaches the maximum at Days 6 to 10, and resolves by Day 14 in patients with vasospasm. ${ }^{3,22)}$ "Delayed" vasospasm is known to occur within 3 weeks after the onset of meningitis. ${ }^{13)}$ Several cases of arteritis are known, but the timing, duration, and recovery are unclear. Arteritis occurred 7 days after bacterial meningitis onset and improved with antibiotic administration. ${ }^{20)}$ Our patient developed arterial narrowing 40 days after meningitis onset, which continued over 6 months and resolved 21 months later. The neuroimaging abnormalities were long lasting compared to previously reported "late onset" cases. ${ }^{15,17)}$ The relationship between the initial transsphenoidal surgery and the meningitis was unclear because of the long interval. Chronic sphenoid sinusitis after transsphenoidal surgery is one possible explanation for late onset of meningitis. A certain inflammatory reaction might be involved, but CSF and blood examination showed no systemic inflammatory reaction. Autoimmune diseases are another possible mechanism. However, this arteritis was thought due to prior bacterial meningitis from the clinical time course. In fact, steroid pulse therapy was not effective. Although the radiographical findings and clinical course in the present case were also similar to atherosclerotic cerebral infarction, follow-up MR angiography showed resolution of the arterial caliber of the left middle cerebral artery (Fig. 2D), so this can be ruled out. Careful diagnostic approach should be considered.

The reasons for the delayed onset and long-lasting course of arteritis, and why the symptoms were so intractable remain unsolved. These situations are not compatible for all considerable entities. Prior history of transsphenoidal surgery might be involved. Moreover, intensive treatment with corticosteroid possibly modified the condition. Further case experience is required.

No treatment has been established for this entity. Triple $\mathrm{H}$ therapy (hypervolemia, hypertension, and hemodilution) is effective for arteritis secondary to tuberculous meningitis, ${ }^{7)}$ and administration of corticosteroid and/or antibiotics is effective. ${ }^{20,23)}$ In our case, the clinical symptoms progressed despite antibiotic administration with hypervolemic therapy, hyperdynamic therapy, and corticosteroid administration. Intrathecal administration of nicardipine was also carried out for possible vasospasm involvement, ${ }^{19)}$ which was also ineffective.

The present case of cerebral arteritis with late onset and prolonged duration, secondary to bacterial meningitis, shows that the natural course of this entity is still unclear and may be extremely intractable. MR plaque imaging is possibly useful for definitive diagnosis.

\section{References}

1) Adams RD, Kubik CS, Bonner FJ: The clinical and pathological aspects of influenza meningitis. Arch Pediatr 65: 354-376, 1948

2) Cairns H, Russell DS: Cerebral arteritis and phlebitis in pneumococcal meningitis. J Pathol Bacteriol 58: 649-665, 1946

3) Chaichana K, Riley LH 3rd, Tamargo RJ: Delayed cerebral vasospasm secondary to bacterial meningitis after lumbosacral spinal surgery: case report. Neurosurgery 60: E206207, 2007

4) Dodge PR, Swartz MN: Bacterial meningitis: a review of selected aspects. II. Special neurologic problems, postmeningitic complications and clinicopathological correla- 
tions. N Engl J Med 272: 1003-1010, 1965

5) Gado M, Axley J, Appleton DB, Prensky AL: Angiography in the acute and post-treatment phases of Hemophilus influenzae meningitis. Radiology 110: 439-444, 1974

6) Greitz T: Angiography in tuberculous meningitis. Acta Radiol Diagn (Stockh) 2: 369-378, 1964

7) Gujjar AR, Srikanth SG, Umamaheshwara Rao GS: HHH regime for arteritis secondary to TB meningitis: a prospective randomized study. Neurocrit Care 10: 313-317, 2009

8) Igarashi M, Gilmartin RC, Gerald B, Wilburn F, Jabbour JT: Cerebral arteritis and bacterial meningitis. Arch Neurol 41: 531-535, 1984

9) Klein IF, Lavallee PC, Touboul PJ, Schouman-Claeys E, Amarenco P: In vivo middle cerebral artery plaque imaging by high-resolution MRI. Neurology 67: 327-329, 2006

10) Küker W, Gaertner S, Nagele T, Dopfer C, Schoning M, Fiehler J, Rothwell PM, Herrlinger U: Vessel wall contrast enhancement: a diagnostic sign of cerebral vasculitis. Cerebrovasc Dis 26: 23-29, 2008

11) Leeds NE, Goldberg HI: Angiographic manifestations in cerebral inflammatory disease. Radiology 98: 595-604, 1971

12) Lehrer $\mathrm{H}$ : The angiographic triad in tuberculous meningitis: A radiographic and clinicopathologic correlation. Radiology 87: 829-835, 1966

13) Moodley M, Bullock MR: Severe neurological sequelae of childhood bacterial meningitis. S Afr Med J 68: 566-570, 1985

14) Pfister HW, Borasio GD, Dirnagl U, Bauer M, Einhaup KM: Cerebrovascular complications of bacterial meningitis in adults. Neurology 42: 1497-1504, 1992

15) Pruitt AA: Central nervous system infections in cancer patients. Neurol Clin 9: 867-888, 1991

16) Raimondi AJ, Di Rocco C: The physiopathogenetic basis for the angiographic diagnosis of bacterial infections of the brain and its coverings in children. I. Leptomeningitis. Childs Brain 5: 1-13, 1979

17) Revest M, Decaux O, Frouget T, Cazalets C, Albert JD, Chevrier S, Guiguen C, Jego P, Grosbois B: [Cryptococcal infections in non-HIV infected patients. Study of four cases and review of literature]. Rev Med Interne 27: 203-208, 2006 (French)

18) Ries S, Schminke U, Fassbender K, Daffertshofer M, Steinke W, Hennerici M: Cerebrovascular involvement in the acute phase of bacterial meningitis. J Neurol 44: 51-55, 1997

19) Suzuki M, Doi M, Otawara Y, Ogasawara K, Ogawa A: Intrathecal administration of nicardipine hydrochloride to prevent vasospasm in patients with subarachnoid hemorrhage. Neurosurg Rev 24: 180-184, 2001

20) Taguchi Y, Takashima S, Noguchi K, Dougu N, Tanaka K: [Cerebral artery lesion in a patient with infective endocarditis: serial MRI and MRA findings of cerebral artery stenosis]. No To Shinkei 58: 879-883, 2006 (Japanese)

21) van de Beek D, de Gans J, Spanjaard L, Weisfelt M, Reitsma JB, Vermeulen M: Clinical features and prognostic factors in adults with bacterial meningitis. N Engl J Med 351: 18491859, 2004

22) Weir B, Grace M, Hansen J, Rothberg C: Time course of vasospasm in man. J Neurosurg 48: 173-178, 1978

23) Younger DS: Vasculitis of the nervous system. Curr Opin Neurol 17: 317-336, 2004

Address reprint requests to: Yoshikazu Ogawa, MD, Department of Neurosurgery, Kohnan Hospital, 4-20-1 Nagamachi Minami, Taihaku-ku, Sendai, Miyagi 982-8523, Japan. e-mail: yogawa@kohnan-sendai.or.jp 